\title{
PLATELET RICH FIBRIN SCAFFOLD COMBINED WITH INDUCED BLOOD CLOT IN PULP REVASCULARIZATION OF NECROTIC IMMATURE PERMANENT TEETH WITH CHRONIC PERIAPICAL LESIONS: A CASE SERIES
}

\author{
Mahmoud M. Saeed ${ }^{1 *}$ M.Sc, Mohamed M. Ibrahim ${ }^{2} P h D$, Sybel M. Moussa ${ }^{3}$ PhD, Nihal A. \\ Leheta ${ }^{4} \mathrm{PhD}$
}

\begin{abstract}
BACKGROUND: Traumatized non-vital permanent teeth with incomplete root formation and divergent apices present particular challenges to clinicians during management with traditional approaches.

OBJECTIVES: This report described the outcomes of pulp revascularization of three traumatized immature necrotic permanent teeth using platelet-rich fibrin scaffold combined with induced blood clot.

MATERIALS AN METHODS: Three patients (two boys aged 8 and 11 years and one girl aged 13 years) were presented with immature maxillary incisors with necrotic pulps. Access cavities were prepared and the root canals were gently irrigated with $1.5 \%$ sodium hypochlorite $(20 \mathrm{~mL} / 5 \mathrm{~min})$. Calcium hydroxide paste was applied three $\mathrm{mm}$ short of the root apex and the canals were temporized with intermediate restorative material. At the following appointment after two weeks, the teeth were treated by revascularization protocol using Platelet rich fibrin scaffold combined with induced blood clot and the three cases were clinically and radiographically followed up to eighteen months.

RESULTS: The patients were clinically asymptomatic at all follow-up periods. Cone Beam Computed Tomography (CBCT) images revealed evidence of root maturation and healing of the periapical lesions. Calcific intra-canal deposits were noted at the middle and apical thirds of the root canals.

CONCLUSIONS: Pulp revascularization procedure using Platelet rich fibrin scaffold showed evidence of root elongation, increased dentin thickness and resolution of the periapical lesions.

KEYWORDS: Immature teeth, Pulp revascularization, Induced blood clot, Platelet concentrates.

RUNNING TITTLE: Platelet rich fibrin in pulp revascularization of non-vital immature teeth.
\end{abstract}

\footnotetext{
1. PhD candidate at the Endodontic Department, Faculty of Dentistry, Alexandria University, Egypt.

2. Professor of Endodontics, Faculty of Dentistry, Alexandria University, Egypt.

3. Professor of Endodontics, Faculty of Dentistry, Alexandria University, Egypt.

4. Lecturer of Endodontics, Faculty of Dentistry, Alexandria University, Egypt.
}

\section{*Corresponding author}

Email: Nivadelsheikh@gmail.com

\section{INTRODUCTION}

Preservation of non-vital permanent teeth incomplete root formation requires advanced technologies, knowledge and expertise (1). The traditional management of necrotic immature teeth involves calcium hydroxide apexification or apical plug with mineral trioxide aggregate (MTA) (2). MTA apical plug requires only single visit and offers a favorable healing response (3), nevertheless the two deficiencies of the Calcium hydroxide apexification, namely the failure to stimulate root maturation and the susceptibility to root fracture, have not yet been improved (4).

Pulp revascularization approach is built on the principle of tissue bio-engineering, which involves the removal of contaminants, the use of three-dimensional scaffolds and bioactive growth factors for progenitor cells recruitment (5). Routinely, periapical bleeding is provoked intentionally during regenerative endodontic therapy, to create an intracanal blood clot. The evoked clot may function as a three-dimensional environment for migration and attachment of endogenous progenitor cells (6).

Platelet concentrates have been introduced to be used as bioscaffolding materials into the root canal (7). The platelet concentrates induce cell differentiation, promote vascular growth, produce anti-inflammatory agents and contain growth factors that improve the potential for wound healing $(7,8)$. The platelet rich fibrin may deliver additional benefits over other platelet concentrates. First, Platelet rich fibrin forms a well-structured fibrin network that contains high concentration of platelets and leukocytes, which might continuously release diverse growth factors (9). Secondly, specific cytokines and essential circulating immune cells in Platelet rich fibrin scaffolds also work against infection. It is therefore reasonable to assume that Platelet-rich fibrin is an ideal bio-scaffold for tooth revascularization (9).

Recent studies have already shown successful and comparable clinical and radiographic outcomes of pulp revascularization technique using platelet concentrates and 
Saeed et al.

evoked blood clot $(8,9)$. However, the outcome of a combined application of evoked blood clot and platelet-rich fibrin has been scarce evidence to date (10). Regenerative endodontics has three treatment outcomes (11); the resolution of the endodontic infection and the evidence of bony healing, improvement in root maturation, and respond positively to pulp vitality tests. Chen et al reported intracanal calcific deposits as one of the healing findings after pulp revascularization approaches (12). Intra-canal calcification and other revascularization outcomes have been reported in several previous studies (12-14).

The goal of this report was to demonstrate the clinical and radiological outcomes of pulp revascularization of three traumatized immature necrotic permanent teeth using platelet-rich fibrin scaffold combined with evoked blood clot.

\section{MATERIALS AND METHODS}

All interventions were carried out in compliance with the institutional ethical standards developed by the IRB of the Faculty of Dentistry, Alexandria University and following informed parent consent.

Three patients (two boys aged 8 and 11 years and one girl aged 13 years) were referred from the pediatric dentistry department to the endodontic department's clinics for endodontic management of immature permanent maxillary lateral and central incisors. All the patients had a history of trauma approximately one year prior to their presentation to the clinic, creating a direct influence on the maxillary incisors. The teeth were not sensitive to percussion or palpation during clinical examination, and did not respond to thermal or electric pulp testing. Sinus tracts were also observed on the labial aspect of the involved teeth. Periodontal examination revealed normal probing depth (2-3 $\mathrm{mm}$ ) around the involved teeth. The preoperative CBCT image for each case revealed an immature tooth with open apex combined with a large radiolucent area. For each case, the sinus tract was traced into the lesion using a Guttapercha cone (size 25). On the basis of clinical and radiographic findings, a common diagnosis of pulp necrosis with asymptomatic chronic apical periodontitis was made for each tooth.

Considering the stage of root development, pulp revascularization was initiated for each tooth using evoked blood clot integrated with Platelet rich fibrin scaffolds after obtaining consent from parents.

Treatment Procedures

The treatment protocol provided for each of the three cases is summed as follows:

At the $1^{\text {st }}$ session: the operative field was anesthetized with 4\% Articaine containing 1:1000.000 Epinephrine (Inibsa, Barcelona, Spain.) and the concerned tooth was isolated using rubber dam (Sanctuary Dental Dam, Malaysia). Dental caries was removed (if present), then the access cavity was prepared and working length periapical radiograph was determined. The root canal was disinfected according to guidelines of the American association of endodontists for regenerative endodontics (15). The root canal was gently irrigated with $1.5 \%$ sodium hypochlorite $(20 \mathrm{ml} /$ canal, 5 min) using a 27- gauge side-vented irrigation needle (Kerr, Switzerland) positioned within two mm of the root apex to minimize extrusion of the irrigant outside the root canal.
Platelet rich fibrin in pulp revascularization of non-vital immature teeth. Minimal instrumentation was done to avoid weakening of the lateral dentinal walls. The root canal was dried with suitable size sterile paper points. Calcium hydroxide paste (UltraCal XS (Ultradent Products, Inc) was applied as intracanal medication two mm short of the root apex and was checked with the periapical radiograph. The access cavity was temporized with thick layer of intermediate restorative material (Dentsply Maillefer, Ballaigues, Switzerland) and the epithelial lining of the sinus tract was removed by a sterile $\mathrm{K}$ file at the end of the visit and swabbed by iodine. The Calcium hydroxide paste was kept in the canal for a period of two weeks.

Second Appointment

Two weeks later, the patient returned back to the endodontic clinic without any symptoms. The involved tooth was asymptomatic to both percussion and palpation tests. The operative field was anesthetized with 3\% Mepivacaine (without vasoconstrictor) and the concerned tooth was isolated using rubber dam. The intermediate restorative material was removed. The Calcium hydroxide paste was entirely flushed from the root canal with copious and gentle irrigation with sterile saline followed by final slow irrigation with $20 \mathrm{ml}$ of $17 \%$ Ethylenediaminetetraacetic acid (Prevest Denpro, EDTA Solution). To expose and extract growth factors from the dentin, ethylenediaminetetraacetic acid was left in the root canal for about one minute and the canal was dried with appropriate size sterile paper points (Figure1A).

A sterile pre-curved size \#30 K-file (Mani Inc.) was introduced $3 \mathrm{~mm}$ beyond the root apex to lacerate the apical tissue and to evoke a blood clot in the canal space just below the cemento-enamel junction (Figure1B).

Simultaneously, platelet-rich fibrin scaffold was prepared by drawing 5-10 cc of the patient's blood from the antecubital vein into non-heparinized test tubes without the addition of an anticoagulant (Figure 2A). The collected blood was instantly centrifuged using a table top centrifuge (800D centrifuge, Makaad, Shanghai, China) at 3000 rpm for 10mins (16) to avoid blood from coagulating after contact with the glass tube. Upon termination of the centrifugation, the platelet-rich fibrin scaffold was removed from the tube with sterile tweezers (Figure 2C\& D). Then it was squeezed in the PRF box to shape it as a membrane (Figure 2E\&F). Using a hand plugger, the Platelet-rich fibrin scaffold (Figure 1C) was applied into the root canal directly over the induced blood clot to a level three mm below the cemento-enamel junction (Figure 1D \&E) and Biodentine cap (Septodont, France) was applied over the platelet-rich fibrin scaffold (Figure 1F). The access cavity was sealed by resin modified glass ionomer cement (RIVA LC, SDI, Australia) (Figure 1G), followed by nanohybrid composite restoration (Z350 XT, 3M ESPE, United States) to provide definitive seal (Figure $1 \mathrm{H})$.

Patient returned to the clinic for clinical and radiographic follow-up evaluation after three, six, twelve, and eighteen months. 
Saeed et al.
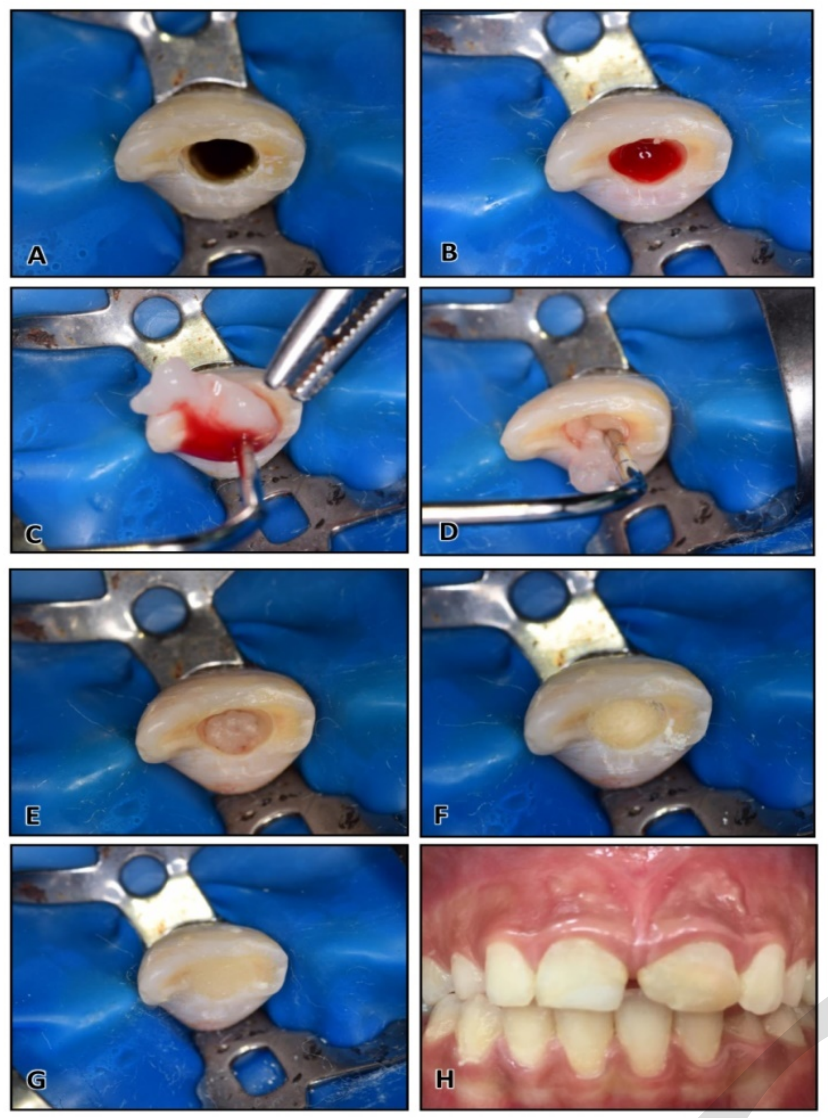

Figure 1: Treatment phase (Second appointment) of a pulp revascularization procedure in a representative case showing: (A) Access cavity preparation for a central incisor after elimination of Calcium hydroxide paste from the canal space, (B) Induction of bleeding inside the canal, (C)\&(D) Application PRF scaffold using a hand plugger, (E) PRF scaffold three mm below the level of CEJ, (F) Placement of Biodentine in the canal directly over the PRF scaffold, (G) Sealing the access cavity with resin modified glass ionomer, (H) Clinical post-operative photograph after final composite resin restoration.
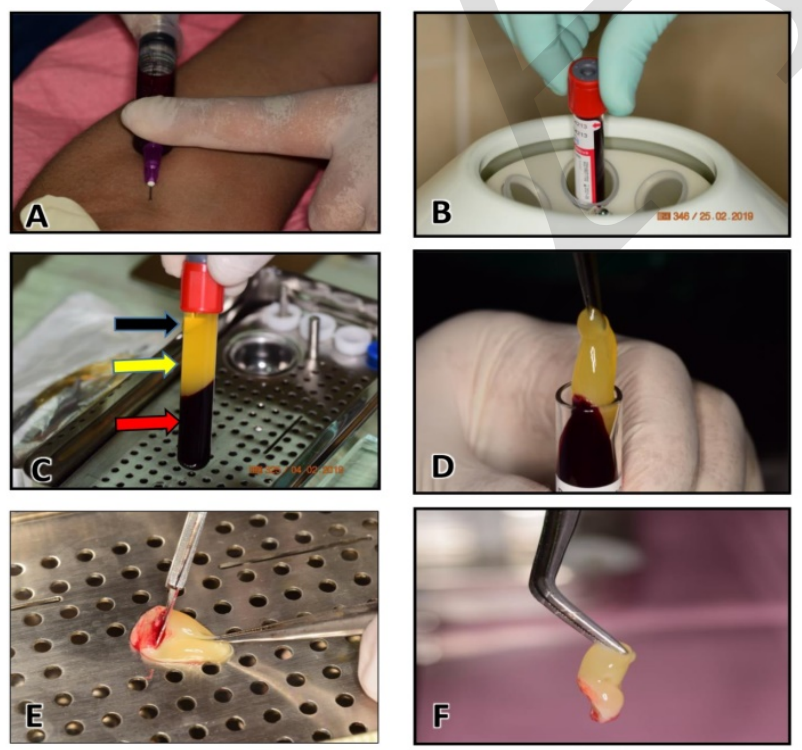

Platelet rich fibrin in pulp revascularization of non-vital immature teeth.

Figure 2: Platelet Rich Fibrin Preparation (A) Ten ml of the patient's entire blood was intravenously drawn from the antecubital vein of the right forearm, (B)The sterile tubes were centrifuged at $2700 \mathrm{rpm}$ for twelve minutes (C) Three layers obtained after centrifugation: platelet-poor plasma at the top (black arrow), platelet-rich fibrin in middle (yellow arrow), and red blood cells at the base (red arrow), (D), (E) \&(F) the PRF clot was extracted and processed in the PRF box.

\section{RESULTS}

The patients were asymptomatic and the teeth were not sensitive to percussion or palpation at all follow-up periods. The treated teeth were not responsive to cold testing with Ethyl chloride spray (Walter Ritter, Germany) and did not respond to electric pulp testing.

Standard periapical digital radiographs were taken for the patients (Figure 3) using a paralleling cone technique with EndoRay aiming device (Dentsply Rinn) which showed initial resolution of the peri-radicular lesion at the threemonth follow-up. Root dentin thickening with gradual apical closure were noted over six-, twelve- and eighteen-months.

Comparing the pre-treatment CBCT images (Morita 3DX; J Morita, Japan) with those obtained after 18 months follow up period, there was evidence of root elongation, narrowing of apical diameter and healing of the periapical lesions with increased bone density. Intra canal calcification with partial obliteration of the root canals was noted. (Figures 4, 5\& 6).
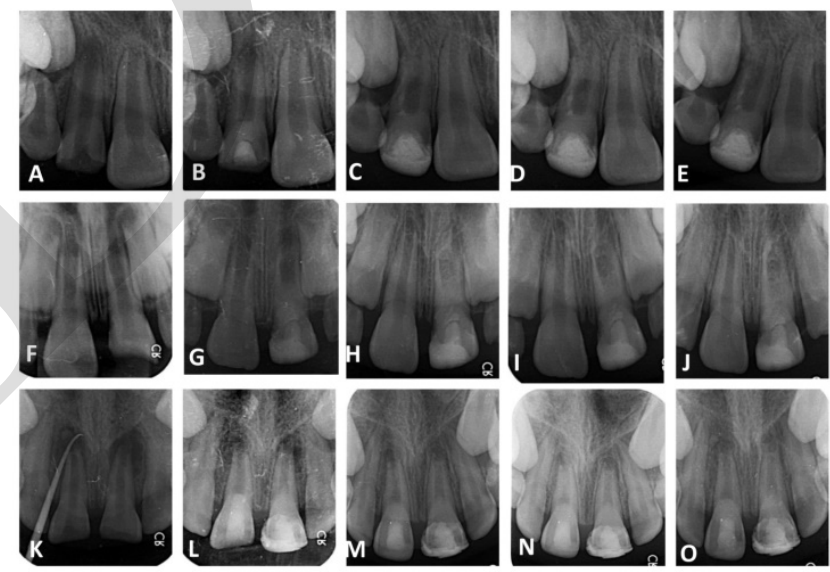

Figure 3:The first row shows the periapical radiographs of the first case (tooth 12): (A) the pretreatment periapical radiograph, (B) three-month post-treatment radiograph, (C) six-month post-treatment radiograph, (D) twelve-month post-treatment radiograph, and (E) eighteen-month posttreatment radiograph. The second row shows the periapical radiographs of the second case (tooth 21): (F) the pretreatment radiograph, $(\mathrm{G})$ three-month radiograph, $(\mathrm{H})$ six-month radiograph, (I) twelve-month radiograph, and (J) eighteen-month post-treatment radiograph. The third row shows the periapical radiographs of the third case (tooth 11): (K) the pretreatment radiograph, (L) three-month radiograph, (M) six-month radiograph, (N) twelve-month radiograph, and (O) eighteen-month post-treatment radiograph. 
Saeed et al.
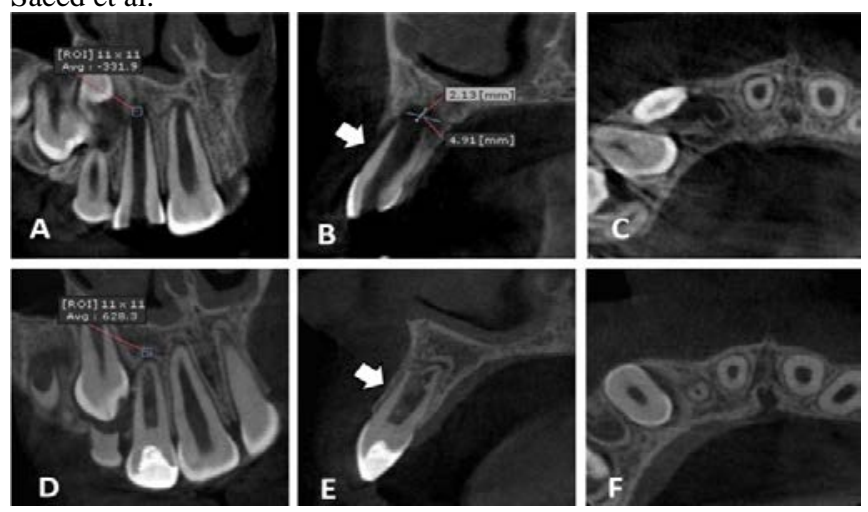

Figure 4: The CBCT 3D images of the first case (tooth 12): (A) A preoperative coronal view showed divergent root apex and the average bone density of the periapical lesion, (B) The sagittal view revealed the dimensions of the periapical lesion and labial bone resorption (arrow), (C) Axial view at the apical third. (D,E\&F) are the 18-month follow-up images, (D) The coronal view reveals progression of root development with apical closure and the average bone density of the periapical area. (E) The sagittal view shows thickening of the dentinal walls with calcific deposits at the apical third and labial bone deposition (arrow). (F) Axial view at the apical third.
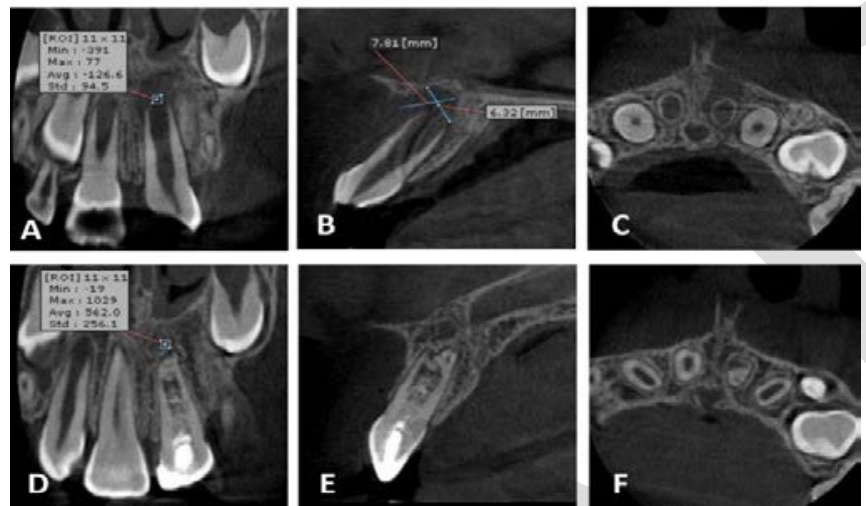

Figure 5: The CBCT images of the second case (tooth 21): (A) A preoperative coronal view revealed an immature tooth and the average bone density of the periapical lesion, (B) The sagittal view revealed the dimensions of the periapical lesion, (C) Axial view at the apical third. (D,E\&F) are the 18-month follow-up images, (D) The coronal view reveals progression of root development with apical closure and the average bone density of the periapical area, (E) The sagittal view shows thickening of the dentinal walls with calcific deposits, (F) Axial view at the level of the apical third.
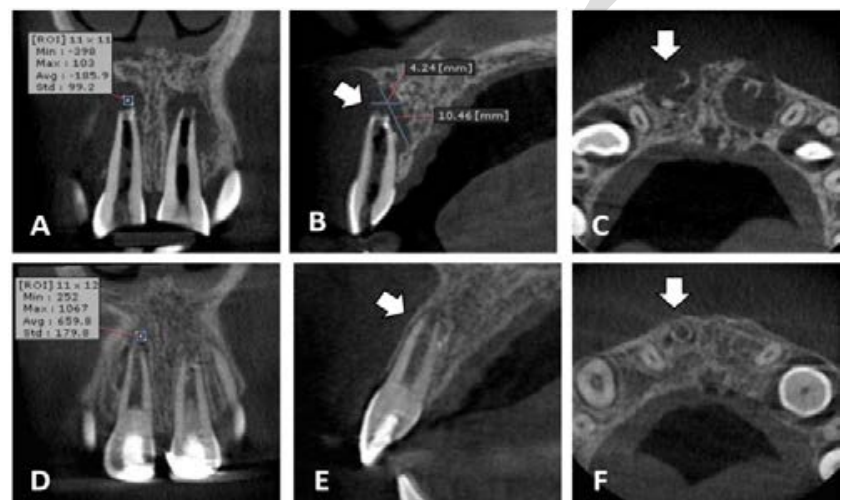

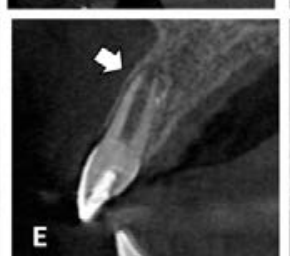

$\mathbf{F}$
Platelet rich fibrin in pulp revascularization of non-vital immature teeth.

Figure 6: The CBCT images of the third case (tooth 11): (A)

A preoperative coronal view revealed an immature tooth and the average bone density of the periapical lesion, (B) The sagittal view revealed the dimensions of the periapical lesion and labial bone resorption (arrow), (C) Axial view at the apical third revealed destruction of labial cortical bone (arrow). (D,E\&F) are the 18-month follow-up images, (D) The coronal view reveals the average bone density of the periapical area (arrow), (E) The sagittal view shows healing of the labial bone, (F) Axial view at the apical third shows healing of the labial bone (arrow).

\section{DISCUSSION}

Several scaffolds have been investigated for use in pulp revascularization protocols $(8,9,12)$. In most pulp revascularization procedures of permanent teeth with non-vital pulp, blood clot is used as a reservoir of essential bio-active growth factors (17). The revascularization techniques that depend on evoked blood clot have been claimed to encourage healing rather than guided pulp regeneration (11). Therefore, the regenerative strategies should utilize suitable scaffolding materials that are not restricted only to revascularize the canal space, but should also promote guided pulp regeneration and enhance other significant events such as odontoblasts and dental nerve fibers differentiation (18). The use of platelet rich fibrin has been recommended as a more suitable bio-scaffold because of its rich content of platelets and leukocytes (19).

Elimination of contaminants from the root canal system is important step in pulp revascularization procedures (20). In the present case series, debridement of necrotic tissue from the root canal was accomplished by carefully irrigating the root canal with $20 \mathrm{~mL} \mathrm{1.5 \%} \mathrm{Sodium}$ hypochlorite and calcium hydroxide paste was used as intracanal medication as recommended by American Association of Endodontists (15). A final irrigation with $17 \%$ Ethylenediaminetetraacetic acid has been recommended prior to lacerating the periapical tissue to promote recruitment of stem cells (21). The advantageous effects of EDTA are mainly attributed to growth factors that are released from dentin and are able to mediate chemotaxis and differentiation of stem cells $(21,22)$.

In the year 2017, Linsuwanont et al demonstrated that root maturation observed in conventional periapical radiographs was not precisely similar to that observed in CBCT of immature permanent teeth with non-vital pulps after pulp revascularization approaches (23). In the present case series, therefore, CBCT was used to determine the preoperative and post-treatment periapical status of the teeth and to assess the stage of root development of the treated teeth, so that a more accurate evaluation could be performed. In the present case series, all revascularized teeth manifested complete resolution of periapical lesions at the end of eighteen months follow-up period, the pre-treatment and post-treatment CBCT images showed a substantial reduction in periapical lesion size and improvement in bone density after eighteen months follow up. The results of the current study regarding healing of the periapical lesions are consistent with the finding presented by Demirci et al in 2020, who utilized CBCT to determine the final periapical status of the teeth after pulp revascularization using blood clot combined with PFF scaffolds (24). They revealed evidence of periapical healing and reduction of the periapical lesions size at the end of the follow-up period. 
Saeed et al.

Evidence of substantial hard tissue deposition was observed in the apical thirds of all treated teeth. The eighteen months follow-up CBCT images demonstrate diverse root maturation patterns including incomplete root maturation and entirely repaired roots with various apical shapes (blunt, cone, irregular). The findings of the present case series were in agreement with Linsuwanont et al in 2017 (23), in their study, they utilized the conventional revascularization protocol suggested by the American Association of Endodontists, they also observed different stages of root maturation with varying apical shapes, which were non-identical to normal mature roots (23).

After eighteen months follow-up, all treated teeth in the present report did not respond to pulp sensibility tests (thermal/electrical). Previous histological studies of animal and human revascularized teeth reported root thickening which was mainly due to formation of bone-like /cementumlike tissues without the histological pattern noticed in dentin $(25,26)$. Even if the afferent fibers close to the recently deposited calcified tissue were regenerated in the root canal, sensitivity tests based on hydrodynamic mechanisms would probably not activate these afferent fibers, since there is no dentinal pattern present in the recently regenerated calcified tissue (27).

Pulp regeneration intends to recapitulate the damaged pulp tissues with all its components, and to restore the morphology and function of the dentin-pulp complex (28). In the current report, intra-canal calcification and calcific deposits were deposited at the root ends. The ability of CBCT to distinguish between dental hard structures (dentine/cementum) and bone has not been verified (23). Previous histological studies demonstrated the deposition of tissues mimicking alveolar bone, cementum and periodontal ligament inside root canal rather than true pulp tissue $(25,26)$, which implicit that pulp regeneration did not occur.

Root canal calcification is a common consequence after pulp revascularization of necrotic immature teeth (12, $13,28)$. In the current case series, intra-canal calcification was found in two treated teeth. The conclusive contributing factors for intra-canal calcific deposits after pulp revascularization are currently unknown. An earlier research by Nosrat et al in 2012 suggested prolonged use of Calcium hydroxide as one of the contributing factors of intra-canal calcification (29). Song et al in 2017 recorded a higher prevalence of intra-canal calcification when periapical bleeding was evoked in the canal compared with cases without induction of bleeding (13), they also observed that intra-canal calcification was higher in calcium hydroxide medicated cases compared with triple antibiotic pastes medicated cases. They concluded that intra-canal calcification is not solely a complication associated with calcium hydroxide but is possibly a complex consequence from multiple contributing factors (13).

\section{CONCLUSIONS}

Pulp revascularization technique using Platelet rich fibrin scaffold integrated with induced blood clot offers a promising therapeutic option for immature teeth with necrotic pulps. More prospective randomized clinical trials and histological studies are necessary to determine the possible healing outcomes after pulp revascularization approaches using PRF scaffolds.
Platelet rich fibrin in pulp revascularization of non-vital immature teeth. REFERENCES

1. Bakhtiar H, Esmaeili S, Fakhr Tabatabayi S, Ellini MR, Nekoofar MH, Dummer PM. Second-generation platelet concentrate (platelet-rich fibrin) as a scaffold in regenerative endodontics: a case series. J Endod. 2017;43:401-8.

2. Yassen GH, Eckert GJ, Platt JA. Effect of intracanal medicaments used in endodontic regeneration procedures on microhardness and chemical structure of dentin. Restor Dent Endod. 2015;40:104-12.

3. Mente J, Hage N, Pfefferle T, Koch MJ, Dreyhaupt J, Staehle HJ, et al. Mineral trioxide aggregate apical plugs in teeth with open apical foramina: a retrospective analysis of treatment outcome. J Endod. 2009;35:1354-8.

4. Cvek M. Prognosis of luxated non-vital maxillary incisors treated with calcium hydroxide and filled with gutta-percha: a retrospective clinical study. Endod Dent Traumatol. 1992;8:45-55.

5. Demarco FF, Casagrande L, Zhang Z, Dong Z, Tarquinio SB, Zeitlin BD, et al. Effects of morphogen and scaffold porogen on the differentiation of dental pulp stem cells. J Endod. 2010;36:1805-11.

6. Hargreaves KM, Giesler T, Henry M, Wang Y. Regeneration potential of the young permanent tooth: what does the future hold? J Endod. 2008;34:51-6 .

7. Mehta S, Watson JT. Platelet rich concentrate: basic science and current clinical applications. J Orthop Trauma. 2008;22:432-8.

8. Murray PE. Platelet-rich plasma and platelet-rich fibrin can induce apical closure more frequently than bloodclot revascularization for the regeneration of immature permanent teeth: a meta-analysis of clinical efficacy. Front Bioeng Biotechnol. 2018;6:139.

9. ElSheshtawy AS, Nazzal H, El Shahawy OI, El Baz AA, Ismail SM, Kang J, Ezzat KM. The effect of platelet-rich plasma as a scaffold in regeneration/revitalization endodontics of immature permanent teeth assessed using 2-dimensional radiographs and cone beam computed tomography: a randomized controlled trial. Int Endod J. 2020;53:90521.

10. Chen YJ, Zhao YH, Zhao YJ, Liu NX, Lv X, Li Q, et al. Potential dental pulp revascularization and odonto/osteogenic capacity of a novel transplant combined with dental pulp stem cells and platelet-rich fibrin. Cell Tissue Res. 2015;361:439-55.

11. Kim SG, Malek M, Sigurdsson A, Lin LM, Kahler B. Regenerative endodontics: A comprehensive review. Int Endod J. 2018;51:1367-88.

12. Chen MY, Chen KL, Chen CA, Tayebaty F, Rosenberg PA, Lin LM. Responses of immature permanent teeth with infected necrotic pulp tissue and apical periodontitis/abscess to revascularization procedures. Int Endod J. 2012;45:294-305.

13. Song M, Cao Y, Shin SJ, Shon WJ, Chugal N, Kim $\mathrm{RH}$, et al. Revascularization-associated intracanal calcification: assessment of prevalence and contributing factors. J Endod. 2017;43:2025-33.

14. Kahler B, Mistry S, Moule A, Ringsmuth AK, Case P, Thomson A, et al. Revascularization outcomes: a 
Saeed et al. prospective analysis of 16 consecutive cases. J Endod. 2014;40:333-8.

15. Law AS. Considerations for regeneration procedures. J Endod. 2013;39(3 Suppl):S44-56.

16. Keswani D, Pandey RK. Revascularization of an immature tooth with a necrotic pulp using platelet-rich fibrin: a case report. Int Endod J. 2013;46:1096-104.

17. Petrino JA, Boda KK, Shambarger S, Bowles WR, McClanahan SB. Challenges in regenerative endodontics: a case series. J Endod. 2010;36:536-41.

18. Piva E, Silva AF, Nör JE. Functionalized scaffolds to control dental pulp stem cell fate. J Endod. 2014;40:S33-40.

19. Choukroun J, Diss A, Simonpieri A, Girard MO, Schoeffler C, Dohan SL, et al. Platelet-rich fibrin (PRF): a second-generation platelet concentrate. Part IV: clinical effects on tissue healing. Oral Surg Oral Med Oral Pathol Oral Radiol Endod. 2006;101:e56-60.

20. Alkahtani A, Alkahtany SM, Anil S. An in vitro evaluation of the cytotoxicity of varying concentrations of sodium hypochlorite on human mesenchymal stem cells. J Contemp Dent Pract. 2014;15:473-81.

21. Casagrande L, Demarco FF, Zhang Z, Araujo FB, Shi S, Nör JE. Dentin-derived BMP-2 and odontoblast differentiation. J Dent Res. 2010;89:603-8.

22. Smith AJ, Duncan HF, Diogenes A, Simon S, Cooper PR. Exploiting the bioactive properties of the dentinpulp complex in regenerative endodontics. J Endod. 2016;42:47-56.

23. Linsuwanont $P$, Sinpitaksakul $P$, Lertsakchai $T$. Evaluation of root maturation after revitalization in immature permanent teeth with nonvital pulps by cone beam computed tomography and conventional radiographs. Int Endod J. 2017;50:836-46.
Platelet rich fibrin in pulp revascularization of non-vital immature teeth.

24. Kandemir Demirci G, Güneri P, Çalışkan MK. Regenerative Endodontic Therapy with Platelet Rich Fibrin: Case Series. J Clin Pediatr Dent. 2020;44:15-9.

25. Wang X, Thibodeau B, Trope M, Lin LM, Huang GT. Histologic characterization of regenerated tissues in canal space after the revitalization/revascularization procedure of immature dog teeth with apical periodontitis. J Endod. 2010;36:56-63 .

26. Martin G, Ricucci D, Gibbs JL, Lin LM. Histological findings of revascularized/revitalized immature permanent molar with apical periodontitis using plateletrich plasma. J Endod. 2013;39:138-44.

27. Saoud TM, Zaazou A, Nabil A, Moussa S, Lin LM, Gibbs JL. Clinical and radiographic outcomes of traumatized immature permanent necrotic teeth after revascularization/revitalization therapy. J Endod. 2014;40:1946-52.

28. Diogenes A, Ruparel NB, Shiloah Y, Hargreaves KM. Regenerative endodontics: A way forward. J Am Dent Assoc. 2016;147:372-80.

29. Nosrat A, Homayounfar N, Oloomi K. Drawbacks and unfavorable outcomes of regenerative endodontic treatments of necrotic immature teeth: a literature review and report of a case. J Endod. 2012;38:1428-34.

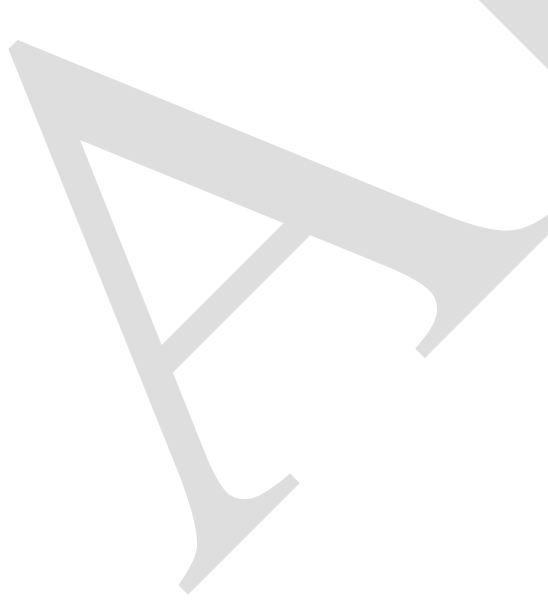

